\title{
Orbital Correlations and Magnetic Phase Transitions in Lightly Doped $\mathrm{La}_{0.88} \mathrm{MnO}_{\mathrm{x}}$ and $\mathrm{LaMn}_{0.94} \mathrm{O}_{\mathrm{y}}$ Manganites
}

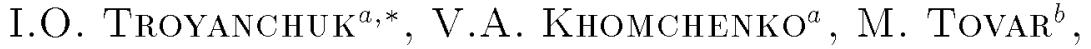 \\ H. SZYMCZAK ${ }^{c}$ AND K. BÄRNER ${ }^{d}$
}

${ }^{a}$ Institute of Solid State and Semiconductor Physics

National Academy of Sciences, P. Brovka str. 17, 220072 Minsk, Belarus

${ }^{b}$ Hahn-Meitner-Institute (BENSC), Glienickerstr. 100, 14109 Berlin, Germany

${ }^{c}$ Institute of Physics, Polish Academy of Sciences

al. Lotników 32/46, 02-668 Warsaw, Poland

${ }^{d}$ IY. Physikalisches Institut der Universität Göttingen

Bunsenstrasse 13-15, 37073 Göttingen, Germany

\begin{abstract}
The results of X-ray and neutron diffraction study as well as the elastic, magnetic, and electrotransport properties of $\mathrm{La}_{0.88} \mathrm{MnO}_{x}(2.82 \leq x \leq 2.96)$ and $\mathrm{LaMn}_{0.94} \mathrm{O}_{y}(2.91 \leq y \leq 3.00)$ manganites have been presented. Under oxygen doping, the transitions from antiferromagnetic to ferromagnetic or spin-glass state are observed for La-deficient and La-excessive series, respectively. These transitions have been found to correlate with the change of the type of Jahn-Teller distortions from static to dynamic ones. The dynamic orbital correlations favor a ferromagnetic state, while an A-type antiferromagnetic state is typical of the static Jahn-Teller distortions. It is argued that the canting magnetic structure in manganites is not realized. The transition to a ferromagnetic or spin-glass state occurs via the formation of inhomogeneous state with different types of orbital correlations and magnetic structure. The key role of intrinsic chemical inhomogeneity in the formation of inhomogeneous magnetic states in solid solutions of manganites is emphasized.
\end{abstract}

PACS numbers: 75.30.Kz, 75.47.Lx, 61.50.Ks

\section{Introduction}

The mixed-valence hole-doped manganites with the general formula $\mathrm{Ln}_{1-x} \mathrm{~A}_{x} \mathrm{MnO}_{3}$ (Ln - lanthanide, $\mathrm{A}$ - alkaline-earth metal) are the model ob-

*corresponding author; e-mail: troyan@ifttp.bas-net.by 
jects of physics of strongly correlated electronic systems. Nature of the interplay between the crystal structure, magnetic and transport properties in these materials is still a matter of discussion in spite of the numerous investigations. Several models were proposed to explain a magnetic state evolution under hole doping as well as the metal-insulator transition at the Curie point. In the double-exchange model of Zener, the simultaneous ferromagnetic and metallic transitions have been qualitatively explained by the fact that electrons tend to move between $\mathrm{Mn}^{3+}$ and $\mathrm{Mn}^{4+}$ ions having the same spin orientation, therefore electron delocalization favors the ferromagnetic order [1]. De Gennes has developed the theory of double exchange and has predicted that a concentration transition from an antiferromagnetic to a ferromagnetic state occurs via the formation of canted homogeneous magnetic structure [2]. More recently Millis et al. pointed out that double exchange alone cannot account for many of the experimental results [3]. The authors showed that a Jahn-Teller type electron-phonon coupling plays an important role in explanation of the colossal magnetoresistance effect and argued the possibility of lattice polaron formation above the temperature of ferromagnetic ordering.

Another mechanism of antiferromagnet-ferromagnet phase transitions in manganites was proposed by Nagaev [4]. Taking into account that the energy of charge carriers in a magnetic semiconductor is minimal for a ferromagnetic state and free charge carriers tend to establish a parallel ordering of the magnetic moments, he predicted that the intermediate phase can be described as non-homogeneous magnetic state driven by electronic phase segregation. In this scenario the ferromagnetic regions contain an excess of holes and are metallic. Antiferromagnetic matrix remains insulating. The transition to metallic conductivity taking place with an increase in doping level occurs when ferromagnetic clusters percolate.

Goodenough et al. argued that the magnetic properties of manganites were determined by the type of orbital state [5]. According to the rules for $180^{\circ}$ superexchange, if the electronic configuration correlates with vibrational modes, $\mathrm{Mn}^{3+}-\mathrm{O}^{2}-\mathrm{Mn}^{3+}$ interactions are antiferromagnetic in the case of the static JahnTeller effect and ferromagnetic when Jahn-Teller effect is dynamic. Thus, antiferromagnetic-ferromagnetic phase transitions can occur through a mixed state of phases with different orbital dynamics.

The existence of phase separation in manganites is corroborated by the numerous experimental data. The results of nuclear magnetic resonance [6], neutron diffraction [7], muon spin relaxation [8], X-ray absorption [9], scanning tunneling spectroscopy [10], electron microscopy [11] experiments give evidence of magnetic and structural inhomogeneities, but the driving force of magnetic phase separation in manganites is still not fully clear. In order to contribute to the solution of this problem we have carried out investigation of antiferromagnetic-ferromagnetic phase transition in low-doped manganites. In the present paper we propose the results of study of structural, magnetic, and electrotransport properties of the 
La-deficient $\mathrm{La}_{0.88} \mathrm{MnO}_{x}$ manganites and compare these results with those obtained for La-excessive $\mathrm{LaMn}_{0.94} \mathrm{O}_{y}$ system.

\section{Experiment}

Polycrystalline samples of $\mathrm{La}_{0.88} \mathrm{MnO}_{x}$ and $\mathrm{LaMn}_{0.94} \mathrm{O}_{y}$ manganites were prepared by solid-state reaction method using high-purity $\mathrm{La}_{2} \mathrm{O}_{3}$ and $\mathrm{Mn}_{2} \mathrm{O}_{3}$ reagents. To remove absorbed water, a prefiring of lanthanum oxide $\left(1000^{\circ} \mathrm{C}\right.$, 1 hour) was carried out. After that, the compacted mixtures of reagents taken in stoichiometric cation ratios were annealed at $950^{\circ} \mathrm{C}$ for $2 \mathrm{~h}$, reground and pressed into pellets. The parent compounds $\mathrm{La}_{0.88} \mathrm{MnO}_{2.96}$ and $\mathrm{LaMn}_{0.94} \mathrm{O}_{3.00}$ were obtained through a final heat treatment at $1300^{\circ} \mathrm{C}$ for $6 \mathrm{~h}$ followed by slow cooling at $30^{\circ} \mathrm{C} / \mathrm{h}$ down to room temperature. The oxygen content of these samples has been determined using thermogravimetric analysis, i.e. decomposition into simple oxides $\mathrm{La}_{2} \mathrm{O}_{3}$ and $\mathrm{MnO}$ in a reducing $\mathrm{H}_{2} / \mathrm{N}_{2}$ flow. The reduction of the samples was carried out in evacuated quartz ampoules at $T=1050^{\circ} \mathrm{C}$ for $24 \mathrm{~h}$ using metallic tantalum. All the samples were quenched. The oxygen loss has been checked by the weighing of the samples before and after reduction. The inaccuracy did not exceed $0.2 \%$ of the total oxygen content. To verify the correctness of determination of the oxygen content in the reduced compounds thermogravimetric analysis was performed for selected samples. In all the cases, results showed good agreement with expected values of the oxygen content. Single-phase compounds of $\mathrm{La}_{0.88} \mathrm{MnO}_{x}$ and $\mathrm{LaMn}_{0.94} \mathrm{O}_{y}$ manganites were obtained in the oxygen concentration ranges $2.82 \leq x \leq 2.96$ and $2.91 \leq x \leq 3.00$, respectively. These ranges correspond to the average valence of manganese ions from 3 to 3.28 for La-deficient series and from 3 to $\sim 3.19$ for La-excessive one. A further reduction was found to lead to decomposition of the samples into $\mathrm{La}_{2} \mathrm{O}_{3}$ and $\mathrm{MnO}$ oxides.

The unit cell parameters as well as the phase purity of the samples were checked by X-ray analysis using a DRON-3 diffractometer with $\mathrm{Cr} K_{\alpha}$ radiation. The refinement of the unit cell parameters has been carried out using the FullProf program. The elastic properties were studied by a resonance method in the sound frequency range $\left(\nu \sim 10^{4} \mathrm{~Hz}\right)$ using cylindrical samples with a diameter of $6 \mathrm{~mm}$ and length of $60 \mathrm{~mm}$. The differential thermal analysis (DTA) was performed at heating (cooling) rates of $10^{\circ} \mathrm{C} / \mathrm{min}$. The measurements of temperature and field dependences of magnetization were carried out with an OI-3001 commercial vibrating sample magnetometer. The study of electrotransport properties was performed using the standard four-probe method using ultrasonically deposited indium contacts. The resistivity data were collected in the temperature range from 80 to $300 \mathrm{~K}$ either in the absence of a magnetic field $(\rho[H=0])$ or in the field of 9 kOe $(\rho[H])$. The magnetoresistance $M R$ has been calculated according to the relation

$$
M R=\{(\rho[H]-\rho[H=0]) / \rho[H]\} 100 \% .
$$


Neutron diffraction measurements for the $\mathrm{La}_{0.88} \mathrm{MnO}_{2.84}$ and $\mathrm{La}_{0.88} \mathrm{MnO}_{2.87}$ samples were performed in the Berlin Neutron Scattering Center using the E9 neutron powder diffractometer (FIREPOD) with a wavelength of neutrons $\lambda=$ $1.7974 \AA$ and scanning step $\Delta \Theta \sim 0.002^{\circ}$. The data have been analyzed with the Rietveld method using the FullProf program.

\section{Results}

According to X-ray diffraction data the $\mathrm{La}_{0.88} \mathrm{MnO}_{x}(2.82 \leq x \leq 2.86)$ samples are characterized by the so-called $\mathrm{O}^{\prime}$-orthorhombic type of unit cell distortion $(b / \sqrt{2}<c<a)$, space group Pnma (Fig. 1). The unit cell values of the most

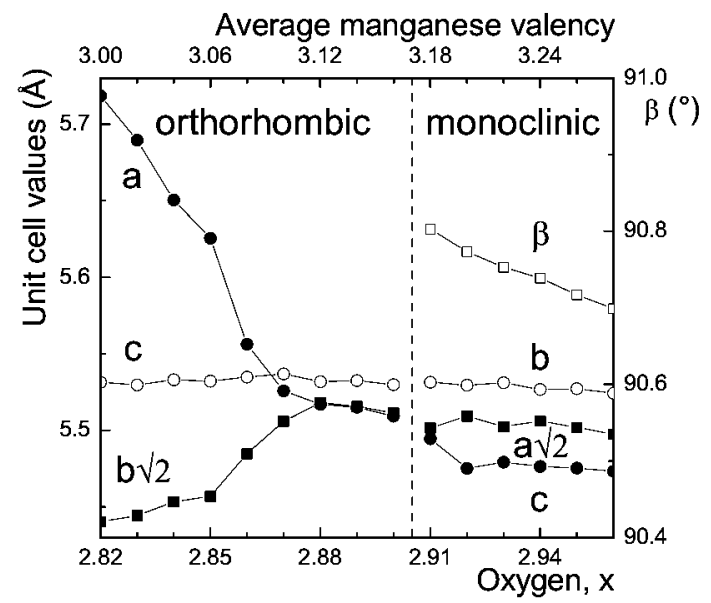

Fig. 1. Room-temperature lattice parameters of $\mathrm{La}_{0.88} \mathrm{MnO}_{x}$ samples as a function of oxygen content.

reduced $\mathrm{La}_{0.88} \mathrm{MnO}_{2.84}$ compound have been found to be very close to those for $\mathrm{LaMnO}_{3}$ stoichiometric composition [12]. The unit cell volume decreases as the oxygen content increases, and the transition to the O-orthorhombic crystal structure $(b / \sqrt{2} \approx a<c)$ occurs starting from $x=2.87$ (Fig. 1). Unlike La $\mathrm{L}_{1-x} \mathrm{~A}_{x} \mathrm{MnO}_{3}$ $\left(\mathrm{A}=\mathrm{Sr}^{2+}, \mathrm{Ba}^{2+}, \mathrm{Pb}^{2+}\right)$ and $\mathrm{LaMnO}_{3+\delta}$ manganites, which exhibit concentration and temperature-induced transitions to rhombohedral unit cell symmetry [13, 14], the compounds $\mathrm{La}_{0.88} \mathrm{MnO}_{x}(2.91 \leq x \leq 2.96)$ go over to monoclinic symmetry (space group $I 2 / a$ ) (Fig. 1). The same structural forms have been found for $\mathrm{LaMn}_{0.94} \mathrm{O}_{y}$ samples (Fig. 2). It is interesting to note that the unit cell parameters of La-deficient manganites are close to those for La-excessive compounds with the corresponding $\mathrm{Mn}^{4+} / \mathrm{Mn}^{3+}$ ratio.

The temperature dependence of Young's modulus for $\mathrm{La}_{0.88} \mathrm{MnO}_{x}$ manganites (square of resonance frequency is proportional to Young's modulus) is presented in Fig. 3. The most reduced $\mathrm{La}_{0.88} \mathrm{MnO}_{2.82}$ sample shows a well-pronounced structural phase transition around $650 \mathrm{~K}$ (Fig. 3a). A large temperature hysteresis 


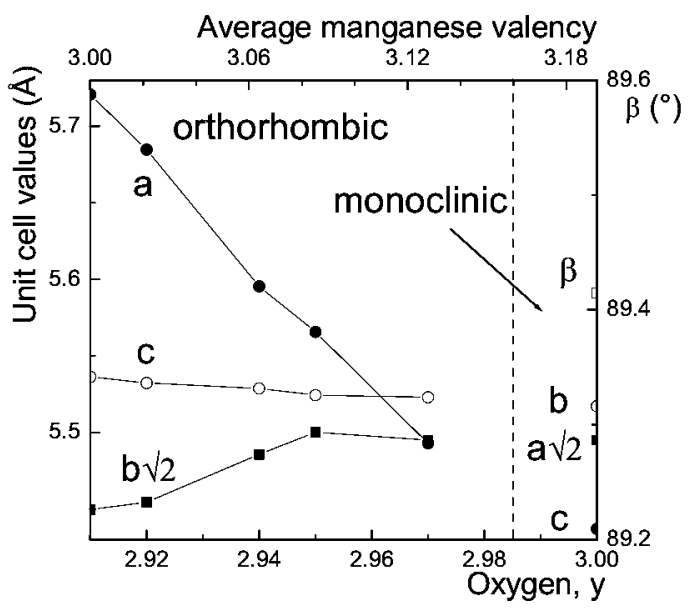

Fig. 2. Room-temperature unit cell parameters of $\mathrm{LaMn}_{0.94} \mathrm{O}_{y}(2.91 \leq y \leq 3.00)$ compounds.
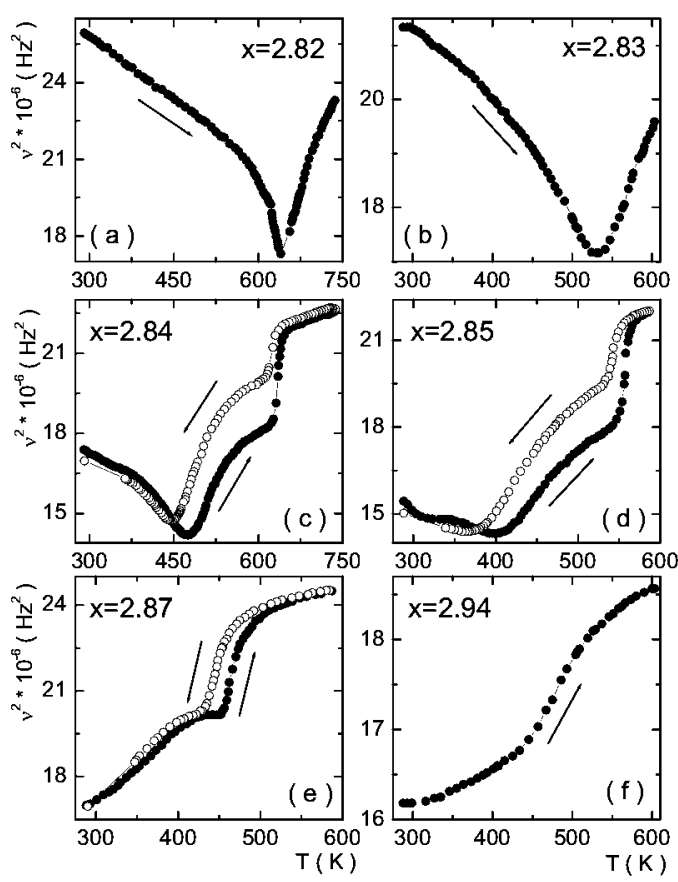

Fig. 3. Temperature dependence of a square of resonance frequency for selected samples of $\mathrm{La}_{0.88} \mathrm{MnO}_{x}$ system. Filled and open symbols are used for measurements on heating and on cooling, respectively.

and strong damping are observed in the vicinity of $650 \mathrm{~K}$. As the oxygen content increases, the temperature of the phase transition lowers gradually and the transition becomes broader (Fig. 3b-d). Besides, a sharp increase in Young's modulus 
indicating the appearance of another structural transition is observed for $x=2.84$ and $x=2.85$ compounds around $630 \mathrm{~K}$ and $530 \mathrm{~K}$, respectively (Fig. $3 \mathrm{c}, \mathrm{d}$ ). The hysteresis observed between heating and cooling runs over a wide range of temperature is evidence of a gradual structural transformation. For the $x=2.86$ compound, the temperature dependence of Young's modulus exhibits a broad minimum slightly above room temperature. The temperature of the second anomaly decreases down to $500 \mathrm{~K}$. In contrast to the $2.82 \leq x \leq 2.86$ samples, no minimum of Young's modulus temperature dependence is observed for the $\mathrm{La}_{0.88} \mathrm{MnO}_{2.87}$ compound above room temperature. However, as in the case of $2.84 \leq x \leq 2.86$ samples, a significant increase in Young's modulus value is observed above $440 \mathrm{~K}$ (Fig. 3e). The monoclinic compounds do not show any sharp changes in the behavior of Young's modulus and exhibit a gradual increase in resonance frequency in the whole accessible temperature range (Fig. 3f).

In order to refine the structural features of $\mathrm{O}^{\prime}$ - and O-orthorhombic samples and to reveal origin of the anomalies observed on the temperature dependence of Young's modulus value we performed neutron diffraction study of $\mathrm{La}_{0.88} \mathrm{MnO}_{2.84}$ and $\mathrm{La}_{0.88} \mathrm{MnO}_{2.87}$ compounds. Diffraction patterns were collected at temperatures of 300 and $530 \mathrm{~K}$ for $x=2.84$, and at $500 \mathrm{~K}$ for $x=2.87$ sample. The

TABLE I

Structural data of $\mathrm{La}_{0.88} \mathrm{MnO}_{2.84}$ and $\mathrm{La}_{0.88} \mathrm{MnO}_{2.87}$ samples at 300,530 , and $500 \mathrm{~K}$, respectively.

\begin{tabular}{|c|c|c|c|c|c|c|c|}
\hline Compound & $T(\mathrm{~K})$ & Cell $(\AA)$ & Atoms & $x$ & $y$ & $z$ & $R$ - factors $(\%)$ \\
\hline \multirow[t]{4}{*}{$x=2.84$} & 300 & $a=5.6275(1)$ & $\mathrm{La} 4 c$ & $0.0353(8)$ & 0.2500 & $0.9944(1)$ & $R_{p}=6.91$ \\
\hline & Pnma & $b=7.7515(9)$ & $\operatorname{Mn} 4 b$ & 0.0000 & 0.0000 & 0.5000 & $R_{w p}=9.22$ \\
\hline & & $c=5.5534(6)$ & $\mathrm{O} 14 c$ & $0.4938(7)$ & 0.2500 & $0.0677(2)$ & $R_{B}=8.51$ \\
\hline & & $\alpha=\beta=\gamma=90^{\circ}$ & $\mathrm{O} 2 \quad 8 d$ & $0.2069(3)$ & $0.5350(9)$ & $0.2285(5)$ & $\chi^{2}=4.94$ \\
\hline$x=2.84$ & 530 & $a=5.5457(6)$ & $\mathrm{La} 4 c$ & $0.0204(4)$ & 0.2500 & $1.0062(1)$ & $R_{p}=6.48$ \\
\hline O-phase & Pnma & $b=7.8563(7)$ & $\mathrm{Mn} 4 b$ & 0.0000 & 0.0000 & 0.5000 & $R_{w p}=8.81$ \\
\hline \multirow[t]{2}{*}{$56.36 \%$} & & $c=5.5663(6)$ & O1 $4 c$ & $0.4925(8)$ & 0.2500 & $0.0705(8)$ & $R_{B}=8.80$ \\
\hline & & $\alpha=\beta=\gamma=90^{\circ}$ & 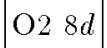 & $0.2250(7)$ & $0.5337(2)$ & $0.2299(7)$ & $\chi^{2}=4.23$ \\
\hline$x=2.84$ & 530 & $a=5.6139(3)$ & La $4 c$ & $0.0365(1)$ & 0.2500 & $0.9960(8)$ & $R_{p}=6.48$ \\
\hline $\mathrm{O}^{\prime}$-phase & Pnma & $b=7.7737(6)$ & $\operatorname{Mn} 4 b$ & 0.0000 & 0.0000 & 0.5000 & $R_{w p}=8.81$ \\
\hline \multirow[t]{2}{*}{$43.64 \%$} & & $c=5.5531(1)$ & $\mathrm{O} 14 c$ & $0.4945(9)$ & 0.2500 & $0.0681(3)$ & $R_{B}=8.88$ \\
\hline & & $\alpha=\beta=\gamma=90^{\circ}$ & O $28 d$ & $0.2047(8)$ & $0.5372(8)$ & $0.2258(1)$ & $\chi^{2}=4.23$ \\
\hline \multirow[t]{4}{*}{$x=2.87$} & 500 & $a=7.8220(8)$ & La $4 e$ & 0.2500 & $0.5023(5)$ & 0.0000 & $R_{p}=4.75$ \\
\hline & $I 2 / a$ & $b=5.5575(2)$ & $\operatorname{Mn~} 4 a$ & 0.0000 & 0.0000 & 0.0000 & $R_{w p}=6.10$ \\
\hline & & $c=5.5043(4)$ & $\mathrm{O} 14 e$ & 0.2500 & $0.0597(7)$ & 0.0000 & $R_{B}=5.42$ \\
\hline & & $\begin{array}{l}\alpha=\gamma=90^{\circ} \\
\beta=90.78(2)^{\circ}\end{array}$ & $\mathrm{O} 28 f$ & $-0.0214(6)$ & $0.2160(9)$ & $0.2781(1)$ & $\chi^{2}=2.21$ \\
\hline
\end{tabular}


TABLE II

Selected interatomic distances (in $\AA$ ) and angles (in degrees) for the $x=2.84$ and $x=2.87$ compounds at different temperatures.

\begin{tabular}{c|l|c|c|c|c|c}
\hline \hline Compound & $T(\mathrm{~K})$ & $\mathrm{Mn}-\mathrm{O} 1(\mathrm{~m})$ & $\mathrm{Mn}-\mathrm{O} 2(\mathrm{~s})$ & $\mathrm{Mn}-\mathrm{O} 2(\mathrm{l})$ & $\mathrm{Mn}-\mathrm{O} 1-\mathrm{Mn}$ & $\mathrm{Mn}-\mathrm{O} 2-\mathrm{Mn}$ \\
\hline$x=2.84$ & 300 & 1.974 & 1.924 & 2.099 & 157.94 & 158.59 \\
& 530 (O-phase) & 2.003 & 1.972 & 2.008 & 157.26 & 161.58 \\
& 530 (O'-phase) & 1.980 & 1.930 & 2.098 & 157.90 & 157.11 \\
\hline$x=2.87$ & 500 & 1.984 & 1.955 & 2.001 & 160.72 & 162.77
\end{tabular}

structural data as well as selected interatomic distances and angles obtained in the Rietveld refinement are presented in Tables I, II. The crystal structure refinement of $\mathrm{La}_{0.88} \mathrm{MnO}_{2.84}$ sample at $T=300 \mathrm{~K}$ was successfully carried out in the space group Pnma using a one-phase structural model (Table I). The $\mathrm{MnO}_{6}$ octahedrons were found to be rather strongly distorted. The bond lengths vary in the range from 1.924 to $2.099 \AA$ (Table II). Such distortions are typical of the static ordering of $d_{z^{2}}$ orbitals. At $530 \mathrm{~K}$, the refinement performed in the one-phase model did not allow to get satisfactory agreement between the observed and calculated diffraction patterns. In the resulting fit, only $\chi^{2}=9.64$ value (with the unit cell parameters $a=5.5627(9) \AA, b=7.8447(5) \AA, c=5.5474(4) \AA)$ was achieved. The agreements factors were significantly improved (Table I) when additional orthorhombic phase was introduced (Fig. 4). According to the obtained results the crystal structure of $\mathrm{La}_{0.88} \mathrm{MnO}_{2.84}$ sample at $T=530 \mathrm{~K}$ is characterized by the coexistence of two orthorhombic phases which differ in oxygen octahedrons distortions. While $\mathrm{MnO}_{6}$ octahedrons in $\mathrm{O}^{\prime}$-phase are strongly distorted indicating

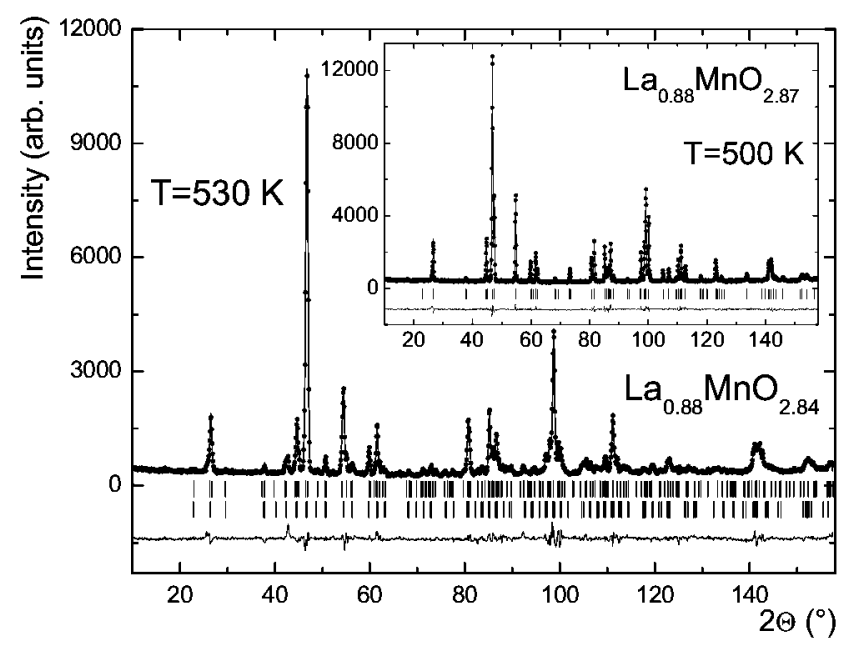

Fig. 4. Observed and calculated powder diffraction patterns of $x=2.84$ and $x=2.87$ (inset) compounds at $530 \mathrm{~K}$ and $500 \mathrm{~K}$, respectively. 
the static $d_{z^{2}}$ orbital ordering, these octahedrons are almost regular for disordered O-phase (Table II). The crystal structure of $\mathrm{La}_{0.88} \mathrm{MnO}_{2.87}$ sample at $T=500 \mathrm{~K}$ was found to be monoclinic. The space group $I 2 / a$ was used for the refinement. The observed and calculated diffraction patterns of the $\mathrm{La}_{0.88} \mathrm{MnO}_{2.87}$ sample at $T=500 \mathrm{~K}$ are shown in the inset of Fig. 4 .

Thus, neutron diffraction data indicate that the minimum on the temperature dependence of Young's modulus value corresponds to the onset of cooperative orbital ordering removal. The two-phase structural state is realized in the temperature range, where hysteresis between heating and cooling runs is observed. A sharp increase in Young's modulus value is associated with the transition from the orthorhombic to monoclinic type of unit cell distortion. The results of both differential thermal analysis and resistivity measurements correlate with the data of neutron diffraction and elastic properties study. The DTA shows a thermal effect at temperatures where the structural phase transitions have been observed. Resistivity measurements carried out for $2.87 \leq x \leq 2.90$ samples above room temperature reveal a hysteresis between the heating and cooling runs in the same temperature range, where elastic and thermal anomalies occur indicating the orthorhombic-monoclinic phase transition.

The magnetic properties reveal a correlation between the magnetic and orbital states. The $\mathrm{La}_{0.88} \mathrm{MnO}_{2.82}$ sample (the lowest oxygen content) has a very small spontaneous magnetic moment (about $0.09 \mu_{\mathrm{B}}$ per manganese ion) and a very large coercive field - $15 \mathrm{kOe}$. The temperature dependence of magnetization for this sample is shown in Fig. 5a. A sharp transition to the paramagnetic state is observed at a temperature of $140 \mathrm{~K}$. An external magnetic field does not shift the temperature of phase transition. An increase in oxygen content in concentration range of $\mathrm{O}^{\prime}$-orthorhombic compositions results in a decrease in Néel point and an increase in spontaneous magnetization. For instance, the spontaneous magnetization of the $x=2.84$ compound is $0.5 \mu_{\mathrm{B}} / \mathrm{Mn}$, the coercive field is $4.2 \mathrm{kOe}$ and the temperature of magnetic ordering is $125 \mathrm{~K}$. The transition into paramagnetic state remains well pronounced. An external magnetic field weakly affects the temperature of transition into paramagnetic state similar to the $x=2.82$ composition. This tendency of the magnetic ordering temperature to decrease remains up to the $\mathrm{La}_{0.88} \mathrm{MnO}_{2.85}$ compound (Fig. 5b). The magnetic state changes with a further increase in oxygen concentration in the range of the orthorhombically distorted samples. Starting from the $x=2.86$ compound, the temperature of magnetic ordering increases from $150 \mathrm{~K}(x=2.86)$ to $202 \mathrm{~K}(x=2.90)$ (Fig. $5 \mathrm{c}$, d). Moreover, a considerable rise of spontaneous magnetization up to $3.25 \mu_{\mathrm{B}} / \mathrm{Mn}$ for $x=2.90$ is observed. Besides, magnetic anisotropy and coercive field decrease significantly. Maximal spontaneous magnetization values (up to $3.5 \mu_{\mathrm{B}} / \mathrm{Mn}$ ) close to that expected for a collinear ferromagnet have been obtained for monoclinically distorted compounds. FC and ZFC curves indicate that the monoclinic samples undergo a paramagnet-ferromagnet transition at temperatures from $212 \mathrm{~K}$ for $\mathrm{La}_{0.88} \mathrm{MnO}_{2.91}$ 

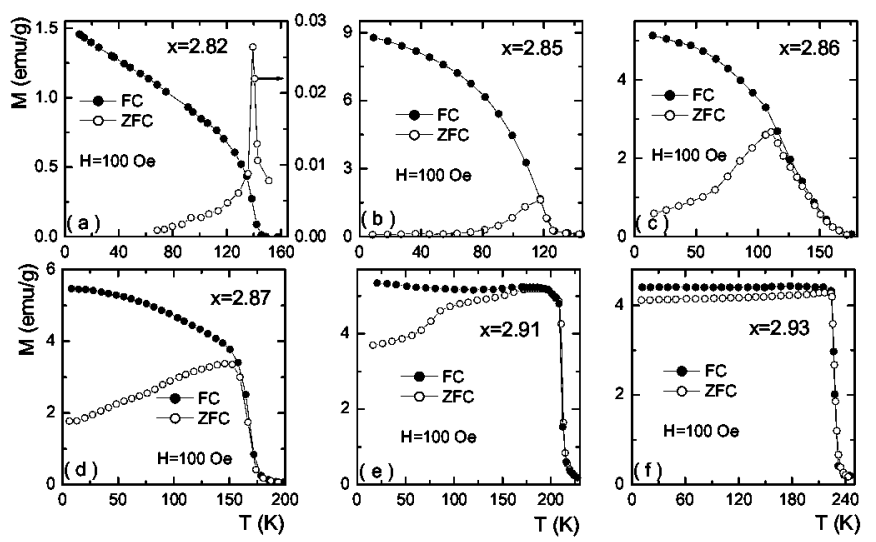

Fig. 5. FC and $\mathrm{ZFC}$ magnetization of selected $\mathrm{La}_{0.88} \mathrm{MnO}_{x}$ compounds, measured at 100 Oe.

to $255 \mathrm{~K}$ for $\mathrm{La}_{0.88} \mathrm{MnO}_{2.96}$ (Fig. 5e, f). In contrast to the $\mathrm{O}^{\prime}$-orthorhombic samples an external field shifts the transition rather far to higher temperatures.

The temperature dependence of resistivity both under zero field or in a field of $9 \mathrm{kOe}$ as well as the magnetoresistance data for selected samples of $\mathrm{La}_{0.88} \mathrm{MnO}_{x}$ system are shown in Fig. 6. In the concentration range $2.92 \leq x \leq 2.96$, the samples are characterized by metallic conductivity below $T_{\mathrm{C}}$ (Fig. 6a). A pronounced magnetoresistance peak is observed slightly below the temperature of the metal-insulator transition. The largest value of the magnetoresistance, up to $96 \%$ at $T=242 \mathrm{~K}$, was found for the $x=2.94$ compound. Starting from the monoclinic $x=2.91$ compound, the semi-conducting type of conductivity is observed (Fig. 6b, c). The magnetoresistance peak at the Curie point gradually decreases with a decrease in oxygen content.
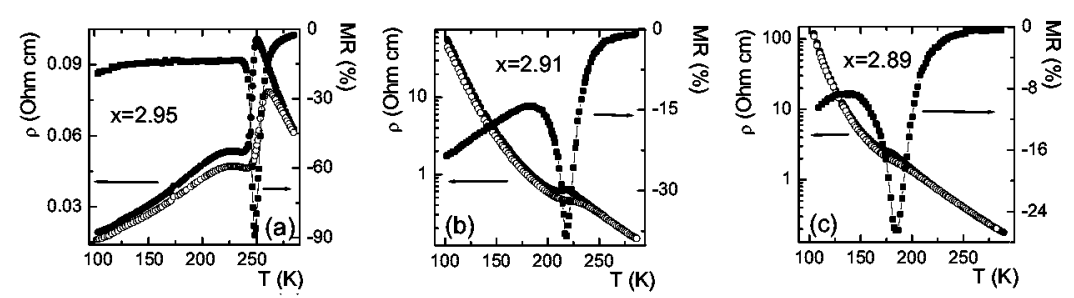

Fig. 6. Temperature dependence of resistivity and magnetoresistance for selected $\mathrm{La}_{0.88} \mathrm{MnO}_{x}$ samples. Filled and open circles are used for the measurements in zero field and an applied field of $9 \mathrm{kOe}$, respectively.

The magnetic properties of the most reduced $\mathrm{LaMn}_{0.94} \mathrm{O}_{2.91}$ sample have been found to be similar to those for the $\mathrm{La}_{0.88} \mathrm{MnO}_{2.82}$ one. The temperature and field dependences of magnetization for La-excessive compounds are shown in Figs. 7, 8. Under oxygen doping, the Néel temperature decreases from $140 \mathrm{~K}$ for $y=2.91$ to $110 \mathrm{~K}$ for $y=2.94$ (Fig. $7 \mathrm{a}-\mathrm{c}$ ). No change of magnetic ordering 

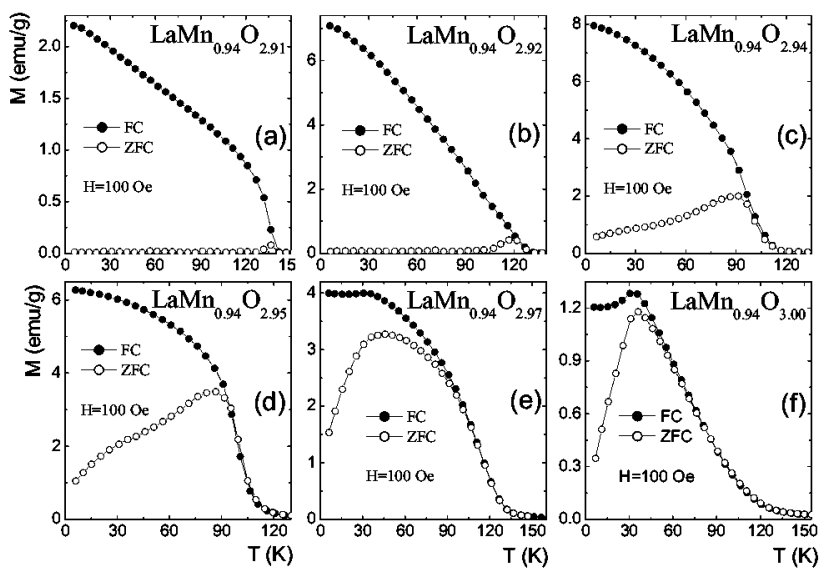

Fig. 7. Temperature dependence of $\mathrm{ZFC}$ and $\mathrm{FC}$ magnetizations for $\mathrm{LaMn}_{0.94} \mathrm{O}_{y}$ $(2.91 \leq y \leq 3.00)$ samples.

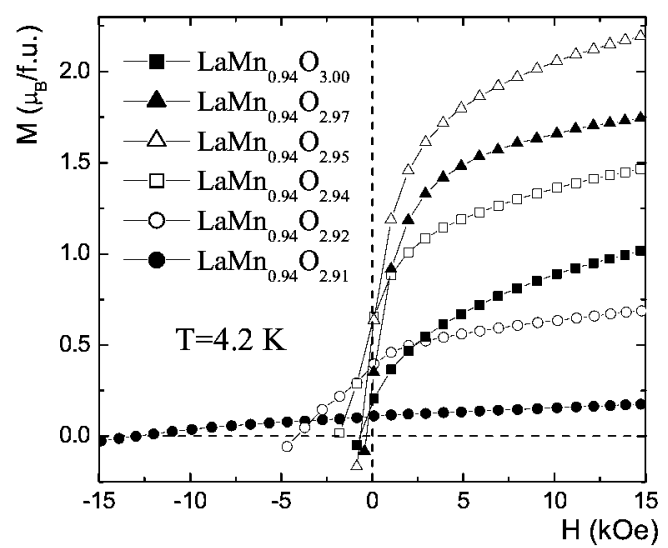

Fig. 8. Field dependence of magnetization for the $\mathrm{LaMn}_{0.94} \mathrm{O}_{y}$ series at $T=4.2 \mathrm{~K}$.

temperature for the $y=2.95$ compound in comparison with the $y=2.94$ sample is observed, but a significant increase in spontaneous magnetic moment from 1 to $1.7 \mu_{\mathrm{B}} / \mathrm{Mn}$ occurs (Figs. $7 \mathrm{~d}, 8$ ). For the O-orthorhombic LaMn $0.94 \mathrm{O}_{2.97}$ compound, the temperature of transition to the paramagnetic state increases to $128 \mathrm{~K}$. At the same time, a slight decrease in spontaneous magnetization value is observed (Fig. 8). Low-temperature behavior of FC and ZFC magnetizations indicates the appearance of a spin-glass component (Fig. 7e). In contrast to La-deficient series, a considerable decrease in spontaneous magnetic moment takes place for the monoclinic $y=3.00$ sample (Fig. 8). The temperature dependence of magnetization of this compound is typical of spin-glasses. Super-paramagnetic behavior is observed below $136 \mathrm{~K}$. The temperature of magnetic moments freezing is about $36 \mathrm{~K}$ (Fig. 7f). All the studied $\mathrm{LaMn}_{0.94} \mathrm{O}_{y}$ compounds are semi-conductors. 


\section{Discussion}

The stoichiometric $\mathrm{LaMnO}_{3}$ is known to be an orbitally-ordered antiferromagnet with the Néel temperature of $140 \mathrm{~K}$ [15]. Cooperative ordering of $d_{z^{2}}$ orbitals observed for this compound is removed around $700 \mathrm{~K}$ as the first-order phase transition [16]. The properties of the most strongly reduced $\mathrm{La}_{0.88} \mathrm{MnO}_{2.82}$ and $\mathrm{LaMn}_{0.94} \mathrm{O}_{2.91}$ samples are similar to the properties of the stoichiometric $\mathrm{LaMnO}_{3}$. Both $x=2.82$ and $y=2.88$ compounds have the same $T_{\mathrm{N}}=140 \mathrm{~K}$ (Figs. 5a, 7a), and exhibit the features of cooperative orbital ordering (Figs. 1, 2, 3a and Ref. [17]). On the other hand, reduced $\mathrm{La}_{0.7} \mathrm{Ca}_{0.3} \mathrm{MnO}_{2.85}$, which also does not contain $\mathrm{Mn}^{4+}$ ions, is a spin-glass with the temperature of magnetic moments freezing around $40 \mathrm{~K}$, it does not show features of cooperative orbital ordering [18]. This results from an oxygen vacancy appearance changing the character of exchange interactions between manganese ions and removing the orbital ordering [18]. Really, $d_{z^{2}}$ orbitals of $\mathrm{Mn}^{3+}$ ions in pentahedron coordination are directed as a rule to oxygen vacancies, which does not agree with cooperative orbital ordering taking into account statistical distribution of oxygen vacancies. Therefore one can believe that oxygen vacancies do not appear in $\mathrm{La}_{0.88} \mathrm{MnO}_{x}$ and $\mathrm{LaMn}_{0.94} \mathrm{O}_{y}$ manganites.

It is well known that the oxygen nonstoichiometry in $\mathrm{LaMnO}_{3+\delta}$ is accommodated through the cation vacancies because the perovskite structure cannot accept excess oxygen in an interstitial site [19]. Accordingly, the composition of $\mathrm{LaMnO}_{3+\delta}$ is expressed as $\mathrm{La}_{1-\varepsilon} \mathrm{Mn}_{1-\varepsilon} \mathrm{O}_{3}$, where $\varepsilon=\delta /(3+\delta)$. Structural defects in La-deficient and La-excessive manganites were studied by neutron diffraction coupled with sample density measurements [20, 21]. It was shown that the defect structure of the sample of nominal composition $\mathrm{La}_{0.91} \mathrm{MnO}_{2.961}$ is realized as $\left[\mathrm{La}_{0.922} \mathrm{Mn}_{0.013}\right] \mathrm{MnO}_{3}$. The resulting distribution model, $\left[\mathrm{La}_{6(1-x)} \mathrm{Mn}_{3(1+x)-V}\right]_{1 /[3(1-x)+V]} \mathrm{MnO}_{3}$, where $x$ is the nominal La-deficiency, and $V$ is the average oxidation state of Mn-ions, is characterized by the conservation of oxygen skeleton. The excess of Mn-ions is located on the La-sites. In such a model, the nominal sample $\mathrm{La}_{0.88} \mathrm{MnO}_{2.82}$ does not contain any vacancies in $\mathrm{A}$-sublattice of $\mathrm{ABO}_{3}$ perovskite and has the cationic composition very close to that for the stoichiometric $\mathrm{LaMnO}_{3}$. Consequently, one could expect that the properties of these two compounds will be similar. Indeed, the both compositions have very close unit cell parameters (Fig. 1), the same spontaneous magnetization value and very close temperatures of magnetic ordering (Fig. 5a) and orbital one (Fig. 3a). Moreover, the proposed model of structural defects is capable to explain the increase in $T_{\mathrm{C}}$ for the compounds $\mathrm{La}_{0.88} \mathrm{MnO}_{x}$ in comparison with $\mathrm{LaMnO}_{3+\delta}$ samples as a result of the conservation of $\mathrm{MnO}_{6}$ octahedrons and the absence of Mn vacancies in B-sublattice.

In the case of La-excessive manganites, the most appropriate structural model seems to be $\mathrm{La}\left[\mathrm{La}_{3-(1-y) V} \mathrm{Mn}_{6(1-y)}\right]_{1 /[(1-y) V+3]} \mathrm{O}_{3}, y$ is the nominal La-excess, and $V$ is the average oxidation state of Mn-ions, where the conser- 
vation of oxygen skeleton and B-site accommodation of excessive La-ions are predicted [20]. Firstly, this model gives an explanation of orbital ordering taking place for the strongly reduced La-excessive compounds (Fig. 2 and Ref. [17]). Secondly, it explains the spin-glass behavior of oxidized samples as a consequence of Mn-vacancies increase observed with increasing oxygen content. On the other hand, according to both Ref. [21] and our neutron diffraction data, the best fitting is observed in the model of the nominal chemical composition. A further research is necessary to reveal the mechanism of nonstoichiometry realization in the $\mathrm{La}_{0.88} \mathrm{MnO}_{x}$ and $\mathrm{LaMn}_{0.94} \mathrm{O}_{y}$ systems. Below we refer to the nominal compositions.

Hypothetical magnetic phase diagrams of the $\mathrm{La}_{0.88} \mathrm{MnO}_{x}(2.82 \leq x \leq 2.96)$ manganites are shown in Fig. 9. The most strongly reduced sample $\mathrm{La}_{0.88} \mathrm{MnO}_{2.82}$ is an antiferromagnet with the Néel temperature of $140 \mathrm{~K}$. With an increase in oxygen content up to the $x=2.85$ sample, the magnetic and orbital ordering temperatures decrease while the spontaneous magnetization increases slightly (Figs. 3, $5 \mathrm{a}, \mathrm{b})$. A further increase in oxygen concentration leads to the appearance of a significant ferromagnetic component. The transition temperature to the paramagnetic state begins to increase and the transition becomes broader (Fig. $5 c, d$ ). The ground state of orthorhombic compounds $2.82 \leq x \leq 2.90$ remains insulating (Fig. 6c). Starting from the $x=2.91$ sample, the monoclinic crystal structure is stabilized (Fig. 1). The appearance of metallic conductivity does not coincide with the transition to the monoclinic phase (Fig. 6b). A metal-insulator transition at $T_{\mathrm{C}}$ (Fig. 6a) is observed for $x \geq 2.92$ compounds.

Traditionally, the evolution of the magnetic properties of manganites under hole-doping, namely, the concentration transition from antiferromagnetic to ferromagnetic state is explained by either the formation of a canted antiferromagnetic structure (double exchange model) [2] or the appearance of ferromagnetic clusters (magnetic phase separation) [4]. The double exchange alone is capable to explain the properties of $\mathrm{La}_{0.88} \mathrm{MnO}_{x}$ manganites only in the limited range of doping concentration where the antiferromagnetic order associates with insulating behavior $(x<2.85)$ and ferromagnetism with metallic conductivity $(x \geq 2.92)$. The double exchange mechanism in itself cannot account for the presence of ferromagnetic insulating phase $2.86 \leq x \leq 2.91$. Recently, theoretical works have focused on investigations of the possibility of magnetic phase separation in manganites. Magnetic phase separation can be driven by electronic [4] or structural phase separation [22]. In the first case, the size of ferromagnetic metallic clusters must be limited by Coulomb interaction [23]. In the case of structural phase separation, even micrometer-size clusters can appear. The experimental data confirm the existence of ferromagnetic clusters with a size up to $100-1000 \AA[11,24,25]$. It is necessary to note that a change from $2 \mathrm{D}$ ferromagnetic interactions characteristic of $\mathrm{LnMnO}_{3}$ to $3 \mathrm{D}$ ones may be induced not only by $\mathrm{Mn}^{4+}$ but also by diamagnetic $\mathrm{Nb}^{5+}[26], \mathrm{Al}^{3+}, \mathrm{Sc}^{3+}[27]$ doping of parent $\mathrm{LnMnO}_{3}$ (Ln-lanthanide). The 


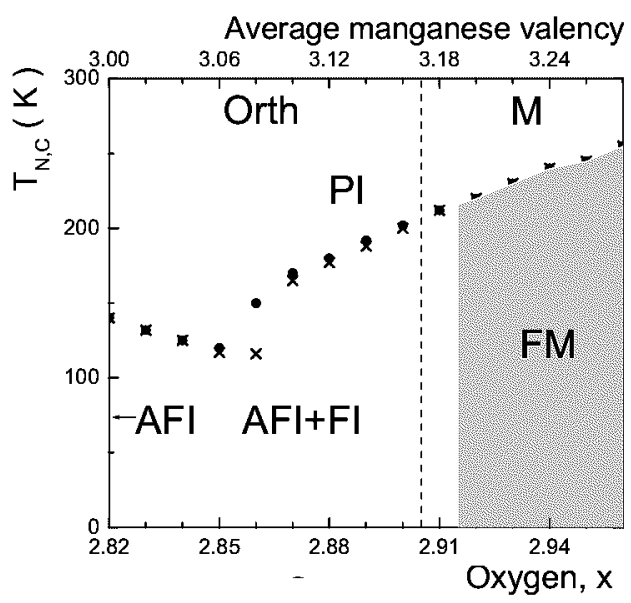

Fig. 9. Magnetic phase diagram of the $\mathrm{La}_{0.88} \mathrm{MnO}_{x}(2.82 \leq x \leq 2.96)$ system. Orth - orthorhombic crystal structure, M - monoclinic crystal structure; PI, AFI, FI, and FM - paramagnetic insulating, antiferromagnetic insulating, ferromagnetic insulating, and ferromagnetic metallic states, respectively. Filled circles present the temperatures corresponding to the onset of magnetic ordering. Cross symbols indicate the temperatures of the most pronounced changes of $\mathrm{FC}$ magnetization measured in 100 Oe (were determined as inflection points on $\mathrm{FC}$ curve).

appearance of ferromagnetic order in spite of the absence of $\mathrm{Mn}^{4+}$ ions contradicts both double exchange and electronic phase separation scenarios. According to Goodenough's consideration, removal of static Jahn-Teller's distortions should lead to the appearance of isotropic ferromagnetic interaction $[5,28]$. In the cases, when one dopes $\mathrm{LnMnO}_{3}$ with non Jahn-Teller ions such as $\mathrm{Mn}^{4+}\left(\mathrm{Nb}^{5+}, \mathrm{Sc}^{3+}\right.$ etc.) the $e_{\mathrm{g}}$ orbitals of all the $\mathrm{Mn}^{3+}$ sites surrounding the $\mathrm{Mn}^{4+}\left(\mathrm{Nb}^{5+}, \mathrm{Sc}^{3+}\right.$ etc.) ion tend to be directed toward the central ion forming an orbital polaron as it was shown in [29]. According to Goodenough-Kanamori rules, an important consequence of such a type of orbital ordering is ferromagnetic exchange interactions between $\mathrm{Mn}^{3+}$ and $\mathrm{Mn}^{4+}$ ions in this cluster. The diamagnetic doping $\left(\mathrm{Nb}^{5+}, \mathrm{Sc}^{3+}\right.$ etc.) also favors ferromagnetism converting 2D-type orbital ordering intrinsic to $\mathrm{LnMnO}_{3}$ into the $3 \mathrm{D}$ cluster-like one with much more fast orbital dynamics. An interaction of ferromagnetic clusters may lead to a long-range ferromagnetic order if $\mathrm{Mn}-\mathrm{O}-\mathrm{Mn}$ angle is enough large. A most likely mixed phase state in $\mathrm{La}_{0.88} \mathrm{MnO}_{x}$ manganites results from the orbital phase separation mechanism. In this scenario the $2 \mathrm{D}$ orbitally ordered clusters are antiferromagnetic (weak ferromagnetic) whereas the 3D quasistatically orbitally ordered ones are ferromagnetic [5]. Apparently, both phases have a slightly different composition. The recent X-ray absorption fine structure measurement of the $\mathrm{Sr}$ and La $K$ edges of the lightly doped solid solutions $\mathrm{La}_{1-x} \mathrm{Sr}_{x} \mathrm{MnO}_{3}$ has revealed the intrinsic chemical and structural inhomogeneity [30]. 


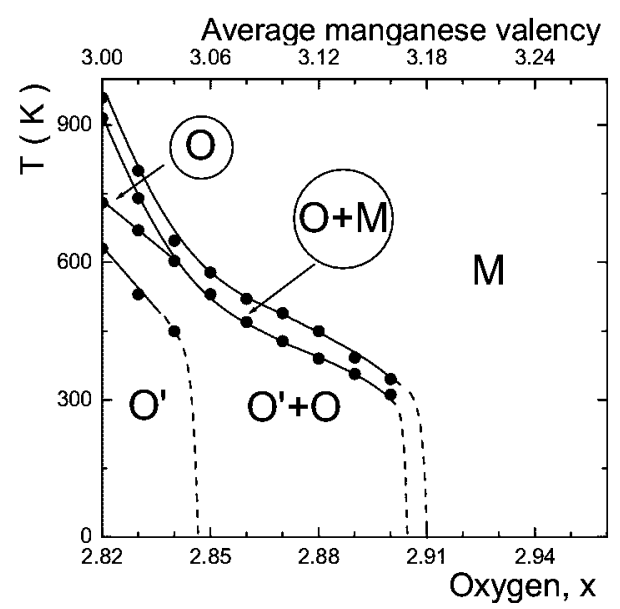

Fig. 10. Hypothetical crystal structure phase diagram of $\mathrm{La}_{0.88} \mathrm{MnO}_{x}$ system. $\mathrm{O}^{\prime}, \mathrm{O}$, and $\mathrm{M}$ - orbitally ordered orthorhombic, orbitally disordered orthorhombic, and monoclinic phases, respectively.

Our study has revealed a strong correlation between the structural and magnetic properties of $\mathrm{La}_{0.88} \mathrm{MnO}_{x}(2.82 \leq x \leq 2.96)$ manganites. The hypothetical structural phase diagram of $\mathrm{La}_{0.88} \mathrm{MnO}_{x}(2.82 \leq x \leq 2.96)$ constructed using $\mathrm{X}$-ray, neutron diffraction, Young's modulus, DTA, and resistivity measurements is shown in Fig. 10. For $\mathrm{La}_{0.88} \mathrm{MnO}_{2.82}$, the sharp drop of Young's modulus value is to be associated with the removal of cooperative orbital ordering and observed at approximately $650 \mathrm{~K}$ (Fig. 3a). The DTA measurements revealed the release of latent heat in the range of $650-730 \mathrm{~K}$, indicating the coexistence of orbitally ordered and orbitally disordered phases up to a temperature of $730 \mathrm{~K}$. Another thermal anomaly connected with the transition to the monoclinic phase is observed in a much narrower temperature range of $915 \leq T \leq 960 \mathrm{~K}$. With an increase in oxygen content to $x=2.83$, the temperatures of both orbital order-disorder and orthorhombic-monoclinic phase transitions significantly decrease. The range of coexistence of $\mathrm{O}^{\prime}$ and $\mathrm{O}$ phases becomes broader, while the width of anomaly associated with temperature-induced orthorhombic-monoclinic transition remains practically constant. Starting from the $x=2.84$ sample, the differential thermal analysis does not show any significant heat effect which could be interpreted as transition to a pure orbitally disordered state. Neutron diffraction data coupled with Young's modulus measurements indicate the existence of orbital ordering at room temperature and two-phase character of the crystal structure above $T \sim 470 \mathrm{~K}$ (Fig. 3c, Table II). A further increase in oxygen concentration leads to the broadening and gradual disappearance of the anomaly which relates to the transition to the orbitally ordered state (Fig. 3d, e). However, the weak hysteresis observed for the $x=2.87$ sample below the temperature of monoclinic-orthorhombic 
phase transition indicates the conservation of orbitally ordered phase (Fig. 3e). It should be noted that the local lattice distortions in $\mathrm{La}_{1-x} \mathrm{Sr}_{x} \mathrm{MnO}_{3}$ manganites are observed even for the metallic compound $x=0.35$ [31]. The temperature of orthorhombic-monoclinic phase transition gradually decreases as the oxygen content increases (Fig. 3c-e), and starting from the $x=2.91$ composition, the monoclinic phase is stabilized (Fig. 1). The coexistence of phases with different crystal structures taking place in a wide concentration and temperature ranges is confirmed by X-ray and neutron diffraction data [14, 32, 33].

Thus, the correlation between the crystal structure and magnetic properties of $\mathrm{La}_{0.88} \mathrm{MnO}_{x}$ manganites is prominent (Figs. 9, 10). The compounds with $x \leq 2.84$ containing significant antiferromagnetic components reveal the features of cooperative orbital ordering, while purely ferromagnetic samples $x \geq 2.92$ are orbitally disordered. Compositions in a wide range of oxygen content $2.85 \leq x \leq 2.91$ exhibit both antiferromagnetic and ferromagnetic components. The broadening of the transition into the paramagnetic state observed for the samples in the intermediate range of doping (Fig. 5b-d) indicates the inhomogeneous magnetic state. Simultaneously, the elastic properties (Fig. 3d, e) suggest the conservation of both orbitally ordered and orbitally disordered phases.

Accordingly, the evolution of magnetic and orbital states in $\mathrm{La}_{0.88} \mathrm{MnO}_{x}$ system is presented as follows. The increase in oxygen concentration above $x=2.82$ value leads to the appearance of holes in the $3 d$ states of $\mathrm{Mn}$ ions (i.e. $\mathrm{Mn}^{4+}$ ions appearance). Orbital ordering is removed nearby $\mathrm{Mn}^{4+}$ ions and the exchange interactions between Mn ions become ferromagnetic. The number of orbitally disordered ferromagnetic clusters increases with an increase in oxygen content. This leads to a lowering of the Néel point (Fig. 5a, b) and an increase in spontaneous magnetization. Simultaneously, the temperature of orbital disordering decreases (Fig. 3a-d). A significant decrease in coercive field and an increase in spontaneous magnetization are observed starting from the $x=2.86$ compound. This denotes a change of the predominant magnetic interaction. X-ray diffraction data (Fig. 1) indicate the removal of cooperative orbital ordering with oxygen content increasing. On the other hand, according to elastic modulus measurements, the local orbital ordering remains at least up to the $x=2.87$ compound (Fig. 3e). With a further increase in oxygen concentration a size of antiferromagnetic phase gradually decreases. The anisotropic contribution concluded from the temperature dependence of the ZFC and FC magnetization for $x \leq 2.91$ samples (Fig. 5a-e) suggests the conservation of antiferromagnetic clusters up to the concentration range of the monoclinically distorted compounds. The value of the spontaneous magnetization of the monoclinic samples $x \geq 2.92$ is consistent with a collinear ferromagnetic state. One could infer that the enhancement of the covalent component of the $\mathrm{Mn}-\mathrm{O}-\mathrm{Mn}$ bond which would occur for orbital-disordered state leads to the crossing of the wide valence and the narrow $3 d$ bands and, starting from the $x=2.92$ compound, the metallic conductivity appears (Fig. 6a). The strong 


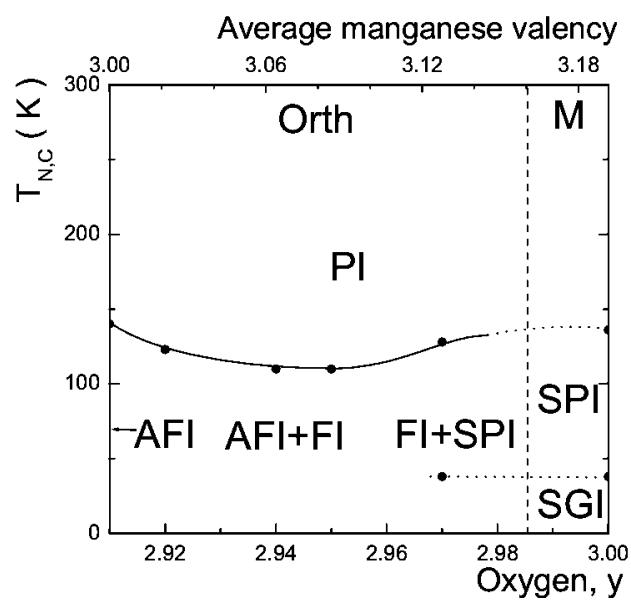

Fig. 11. Magnetic phase diagram of the $\mathrm{LaMn}_{0.94} \mathrm{O}_{y}(2.91 \leq y \leq 3.00)$ system. Orth - orthorhombic crystal structure, M - monoclinic crystal structure; PI, AFI, FI, SGI, and SPI - paramagnetic, antiferromagnetic, ferromagnetic, super-paramagnetic, and spin-glass insulating states, respectively.

dependence of the ferromagnetic order on the external magnetic field results in the colossal magnetoresistance effect near the Curie temperature.

The similar scenario seems to be realized for La-excessive series, but an increase in Mn-ions vacancies observed with increasing oxygen content suppresses the long-range magnetic ordering, and the spin-glass phase for the most oxidized compounds is stabilized (Fig. 11).

\section{Conclusions}

The study of crystal structure, magnetic and electrotransport properties of $\mathrm{La}_{0.88} \mathrm{MnO}_{x}(2.82 \leq x \leq 2.96)$ and $\mathrm{LaMn}_{0.94} \mathrm{O}_{y}(2.91 \leq y \leq 3.00)$ manganites was carried out. The most reduced $x=2.82$ and $y=2.91$ samples which contain only $\mathrm{Mn}^{3+}$ ions were shown to be orbitally ordered antiferromagnets with $T_{\mathrm{N}}=140 \mathrm{~K}$. Their properties are very similar to those of the $\mathrm{LaMnO}_{3}$ stoichiometric compound. The crystal structure of La-deficient and La-excessive samples was found to undergo a number of concentration transitions from orbitally ordered orthorhombic to orbitally disordered orthorhombic and then to monoclinic type of unit cell distortions. As oxygen content increases, the transitions from antiferromagnetic to ferromagnetic or spin-glass state were observed for La-deficient and La-excessive series, respectively. It was shown that the magnetic properties of investigated samples strongly correlate with the type of their orbital state. The compounds with an antiferromagnetic component exhibit the features of cooperative orbital ordering, while ferromagnetic or spin-glass samples are orbitally disordered. The antiferromagnet-ferromagnet phase transition was described in 
the framework of a structurally driven magnetic phase separation model taking into account the key role of intrinsic structural inhomogeneities.

\section{Acknowledgments}

The work was partly supported by Belarus Fund for Basic Research (Projects F01-022 and F03-102), NATO grant PST.EV.979354, the State Committee for Scientific Research (project 5P03B01620), Nanotechnology program (Nanotechnology 3.3), and German Scientific Fund (DFG).

\section{References}

[1] C. Zener, Phys. Rev. 82, 403 (1951).

[2] P.G. De Gennes, Phys. Rev. 118, 141 (1960).

[3] A.J. Millis, B.I. Shraiman, R. Mueller, Phys. Rev. Lett. 77, 175 (1996).

[4] E.L. Nagaev, Phys. Rep. 346, 387 (2001).

[5] J.B. Goodenough, A. Wold, R.J. Arnott, N. Menyuk, Phys. Rev. 124, 373 (1961).

[6] G. Allodi, R. De Renzi, G. Guidi, Phys. Rev. B 57, 1024 (1998).

[7] M. Hennion, F. Moussa, G. Biotteau, J. Rodriguez-Carvajal, L. Pinsard, A. Revcolevschi, Phys. Rev. Lett. 81, 1957 (1998).

[8] R.H. Heffner, L.P. Le, M.F. Hundley, J.J. Neumeier, G.M. Luke, K. Kojima, B. Nachumi, Y.J. Uemura, D.E. MacLaughlin, S.-W. Cheong, Phys. Rev. Lett. 77, 1869 (1996).

[9] C.H. Booth, F. Bridges, G.H. Kwei, J.M. Lawrence, A.L. Cornelius, J.J. Neumeier, Phys. Rev. Lett. 80, 853 (1998).

[10] M. Fath, S. Freisem, A.A. Menovsky, Y. Tomioka, J. Aarts, J.A. Mydosh, Science 285, 1540 (1999).

[11] M. Uehara, S. Mori, C.H. Chen, S.-W. Cheong, Nature 399, 560 (1999).

[12] J. Rodriguez-Carvajal, M. Hennion, F. Moussa, A.H. Moudden, L. Pinsard, A. Revcolevschi, Phys. Rev. B 57, R3189 (1998).

[13] A. Urushibara, Y. Moritomo, T. Arima, A. Asamitsu, G. Kido, Y. Tokura, Phys. Rev. B 51, 14103 (1995).

[14] C. Ritter, M.R. Ibarra, J.M. De Teresa, P.A. Algarabel, C. Marquina, J. Blasco, J. Garcia, S. Oseroff, S.-W. Cheong, Phys. Rev. B 56, 8902 (1997).

[15] E.O. Wollan, W.C. Koehler, Phys. Rev. 100, 545 (1955).

[16] N.V. Kasper, I.O. Troyanchuk, J. Phys. Chem. Solids 57, 1601 (1996).

[17] J. Topfer, J.B. Goodenough, Chem. Mater. 9, 1467 (1997).

[18] I.O. Troyanchuk, S.V. Trukhanov, H. Szymczak, J. Przewoznik, K. Bärner, JETP 93, 161 (2001).

[19] J.A.M. Van Roosmalen, E.H.P. Cordfunke, R.B. Helmholdt, W.H. Zandbergen, J. Solid State Chem. 110, 100 (1994).

[20] R. Horyn, A. Sikora, E. Bukowska, J. Alloys Comp. 353, 153 (2003). 
[21] M. Wolcyrz, R. Horyn, F. Bouree, E. Bukowska, J. Alloys Comp. 353, 170 (2003).

[22] E. Dagotto, T. Hotta, A. Moreo, Phys. Rep. 344, 1 (2001).

[23] L.P. Gor'kov, A.V. Sokol, JETP Lett. 46, 333 (1987).

[24] A.M. Balagurov, V.Yu. Pomjakushin, D.V. Sheptyakov, V.L. Aksenov, N.A. Babushkina, L.M. Belova, O.Yu. Gorbenko, A.R. Kaul, Eur. Phys. J. B 19, 215 (2001).

[25] S. Mori, C.H. Chen, S.-W. Cheong, Nature 392, 473 (1998).

[26] I.O. Troyanchuk, M.V. Bushinsky, H. Szymczak, K. Bärner, A. Maignan, Eur. Phys. J. B 28, 75 (2002).

[27] J.B. Goodenough, R.I. Dass, J. Zhou, Solid State Sciences 4, 297 (2002).

[28] J.-S. Zhou, H.Q. Yin, J.B. Goodenough, Phys. Rev. B 63, 184423 (2001).

[29] T. Mizokawa, D.I. Khomskii, G.A. Sawatzky, Phys. Rev. B 63, 024403 (2000).

[30] T. Shibata, B. Bunker, J.F. Mitchel, P. Schiffer, Phys. Rev. Lett. 88, 207205 (2002).

[31] D. Louca, T. Egami, E.L. Brosha, H. Roder, A.R. Bishop, Phys. Rev. B 56, R8475 (1997).

[32] B.B. Van Aken, O.D. Jurchescu, A. Meetsma, Y. Tomioka, Y. Tokura, T.T.M. Palstra, Phys. Rev. Lett. 90, 066403 (2003).

[33] B. Dabrowski, R. Dybzinski, Z. Bukowski, O. Chmaissem, J.D. Jorgensen, J. Solid State Chem. 146, 448 (1999). 